$\begin{array}{ll}\text { Research Square } & \text { Preprints are preliminary reports that have not undergone peer review. } \\ \text { They should not be considered conclusive, used to inform clinical practice, } \\ \text { or referenced by the media as validated information. }\end{array}$

\title{
Interactome, Network, and Enrichment Motif Analysis Reveals Possible Linkage Between Human Mental Disorders, Emotions, and Coronavirus: In- Silico Insight
}

Peter T. Habib ( $\sim$ p.habib911@gmail.com )

Colors Medical Laboratories

\section{Research Article}

Keywords: Keywords: Motifs, Transcription Factors, Protein-Protein interaction

Posted Date: March 29th, 2021

DOI: https://doi.org/10.21203/rs.3.rs-368781/v1

License: (9) This work is licensed under a Creative Commons Attribution 4.0 International License. Read Full License 


\section{Abstract}

The pandemic of COVID-19 has caused a global crisis. Today, everybody focuses on COVID-19 infection prevention, preparation, and discussion of physical health effects issues. It is important to understand, however, that a few will face life-threatening negative effects on physical health, but that all people will face the negative impact of the pandemic on mental health. COVID-19 hospitals are established in different locations to address the physical health implications of the pandemic. However, it is necessary to understand the effects of infections on mental health more effectively to prevent the negative consequences of infection. Here, we try to find out how the infection could affect mental health. We identify motifs in SARS-CoV-2 that are predicted to interact with human transcription factors (TF). Those TFs regulating behavior and mental health. Our results show that SARS-CoV-2 infection may lead to overactivation or inhibition of critical genes already known to affect behavior and mental health. This study is still limited to in silico limits so, clinical investigation needs to be addressed to assess our hypothesis.

\section{Introduction}

The 2019 new Coronavirus (2019-nCoV) or Severe Corona Virus 2 (SARS-CoV-2) acute respiratory syndrome, as it is currently known, has rapidly expanded from its origins in China Wuhan to the rest of the world. Around 126,841.807 cases (COVID-19) of coronavirus disease 2019 and 2,782,099 cases (Worldometers.info/Coronavirus/) were reported until 27/03/2021. Scientists over the world exhibited all efforts to understand and find a drug-using repurposing approach (1-4), utilizing machine learning and artificial intelligence for more understanding(5-9), and design in silico vaccine (10-13). The pandemic outbreak of SARS-CoV-2 or COVID-19 caused a disaster and caused a sense of serious insecurity and panic $(14,15)$. The mass media/telecommunications /newspaper/blogs have been updating the information about the rapid rise in cases leading to admission to the hospital, being kept in the isolation ward, requiring oxygen support, being admitted in intensive care units (ICUs), associated mortality and the fate of the dead bodies (packaging/filled up graveyards/crematorium). All this news has resulted in significant public fear, anxiety, uncertainty, and unrest. In this context, the diagnosis gives you a sense of shock and disbelief, as well as the feeling that you are on the bed of your death when you are diagnosed with a COVID-19 infection. Many times the diagnosis leads both to hospital entry and a diagnosis of COVID-19 infection in other members of the family, to quarantine for family, and to other contacts (16).

COVID-19 is considered to have major negative mental health effects, not only in those afflicted with the infection and their family members but also in the general population, owing to its high infectivity and observed consequences of infections, including mortality (17-19) and the front line health care workers (HCWs) (20-23). Mental health experts' views on the many emotional reactions/issues and potential psychological disorders that could occur in individuals living with COVID infection are currently available $(24,25)$. Because of the negative mental health implications, it is recommended that a mental health specialist be included in the central management committee of patients with COVID-19 infection (24).

During the post-illness/recovery period, patients admitted with SARS (2002) and Middle East Respiratory Syndrome (MERS, 2012) have been shown to have post-traumatic stress disorder, depression, and anxiety disorders, according to available literature from past infectious disease epidemics (26). Furthermore, new research suggests that delirium may occur (confusion, agitation, altered consciousness) Displacement of neuropsychological deficiencies (dysexecutive syndrome) for patients admitted to ICU with serious COVID-19 infection $(27,28)$.

Many topics investigated the link between mood or emotions and protein-protein interaction $(29,30)$. So far, Researchers that have used mice to demonstrate that, following a viral infection, brain cells that line the blood vessels produce a protein that impairs neuronal fire in the brain area essential for learning, memory, and mood are well studied in the interaction with protein emotions. Researchers say that blocking this protein will at least remove the early stages of illness behavior. (31).

However, the psychological experience of COVID-19 patients during their hospital stay is very minimal. In one study $(n=144)$, the second study ( $n=26)$ recorded high levels of anxiety and depression (34 percent), respectively after an extensive week of hospitalization and HAM-A and HAM-D, respectively, after one week of hospitalization, (Yang et al., 2020). The prevalence of depression was found by another study $(n=57)$ to be about 30 percent recovered COVID-19 patients (32). Moreover, 96.2 percent have recorded post-traumatic stress signature in the large samples $(n=714)$ of hospitalized $(33)$. These researches, however, had not studied detailed the emotional responses of patients before discharge in the hospital. Here we try to explore the link between coronavirus and behavioral genes through a profound analysis of the coronavirus motif and the interaction between human and gene transcription factor activated by the gene.

\section{Materials And Methods}

547 Behavioral genes in protein FASTA format obtained from uniport reviewed (Swiss-Prot) - Manually annotated database, and SARS-COV-2 genes from NCBI in protein FASTA format. The pipeline of research was carried out using various online bioinformatics methods and is made up of four key steps (Figure 1) (34).

A MEME-ChIP analysis (http://meme-suite.org/to) performed on a wide set of sequences associated with chip-Seq or clip-seq human DNA experiments performed a systematic motif analysis (including the finding of motifs) of the entire coronavirus.

Another method of the MEME suite, the Tomtom query (http://meme-suite.org/doc/tomtom.html) was used to compare the motifs against a database of wellknown motifs (i.e., HOCOMOCO human v11 full). Hocomoco is a complete set with a large ChIP-Seq study of transcription binding factor models to humans. In the database, Tomtom classified the motifs and generated alignment for any significant match by searching one or more query motifs for one or more 
human target motif databases (and their reverse complements when applicable). The report was a list of objectives classified by p-value in order to display the queries in the input file for each query. Also reported were the E-value and the Q-value for each match. The q-value is the minimum inaccurate rate at which the similarity observed is considered meaningful. Using the Benjamini and Hochberg procedure, Tomtom evaluated q values of all the corresponding $\mathrm{p}$-values. The meaning was measured by default by the match q-value (35). A list of TFs containing the commonly maintained domain was obtained for all reasons queries.

STITCH, a web tool for defining the most important connection within query TFs, was added to the list of the TF's (http://stitch.embl.de/). A broad database of functional interaction networks from many species is used for the bioinformatics method, and each linked TF is traceable to a source network used to predict it (36).

Protein-protein (PPI) interactions have been conducted for a more detailed investigation. Nearly every phase in live cells is mediated by PPI. The discovery of new PPIs is therefore the key to understanding the biological system's complexity. Several test methods have been developed to classify new PPIs, including yeast purification two hybrids, and tandem affinities. We used ILoops, which predict whether two proteins will interact with structural characteristics (SFs). The aim is to use PPI and non-interacting protein pairs (NIPs)-studied loops or domain patterns to predict binding between protein pairs. Prediction of all compartmental genes and all genes of SARS-COV-2.

\section{Results And Discussion}

The results of our in silico analysis allowed the identification of specific motifs on the SARS-COV-2 able to bind and, therefore, according to our hypothesis, to sequester 139 Transcription Factor (TFs). Using STITCH analysis, 109 of those TFs were connected in a gene regulatory knowledge network, while thirty seemed to be out of this network or had no interactions (Figure 2).

This in silico approach allowed us to predict the recruitment of some TFs directly related to different clinical manifestations (some of them syndromic) characterized y growth retardation, dysmorphic features, intellectual disability, and others. STITCH provides the option of filtering the node related to the biological process. Although there are more than three hundred genes in the network, we tried to go deeper in five genes shared between Iloops and STITCH out of nine responsible for behavior produced by STITCH shown in (Figure 3).

\section{FOXP2}

It is expressed in the fetal and adult brain as well as in several other organs such as the lung and gut. The protein product contains a FOX DNA-binding domain and a large polyglutamine tract and is an evolutionarily conserved transcription factor, which may bind directly to approximately 300 to 400 gene promoters in the human genome to regulate the expression of a variety of genres. Foxp2 was found to influence emotional vocal expressions, which can be investigated in future experiments using selective knockdown of Foxp2 in specific brain circuits $(37,38)$. Genetic studies have associated FOXP2 variation with speech and language disorders and other neurodevelopmental disorders (NDDs) involving pathology of the cortex. In this brain region, FoxP2 is expressed from development into adulthood, but little is known about its downstream molecular and behavioral functions. FOXP2 is a brain-expressed transcription factor implicated in a rare disorder involving speech apraxia and language impairments. cortex-specific Foxp2 conditional knockout mice and found a major deficit in reversal learning, a form of behavioral flexibility $(39,40)$. and inhuman $(41)$. Speech difficulties lead to difficulty in producing words

$(42,43)$ Nevertheless, scientists identified the roles of Foxp2 in skull shaping and bone remodeling. They indicate that Foxp2 helps to regulate the strength and length of hind limbs and maintenance of joint cartilage and intervertebral discs, which are all anatomical features that are susceptible to adaptations for bipedal locomotion. Considering the known roles of Foxp2 in brain circuits that are important for motor skills and spoken language, the researchers suggest that this gene may have been well placed to contribute to the coevolution of neural and anatomical adaptations related to speech and bipedal locomotion. (44). Moreover, Cell type-specific gene expression profiling of cortical pyramidal neurons revealed aberrant regulation of genes involved in social behavior. In particular Foxp2 mutants showed the downregulation of Mint2 (Apba2), a gene involved in approach behavior in mice and autism spectrum disorder in humans. Taken together these data demonstrate that cortical Foxp2 is required for normal social behaviors in mice (41).

\section{MEF2C}

Is a transcription activator that binds specifically to the MEF2 element present in the regulatory regions of many muscle-specific genes. Controls cardiac morphogenesis and myogenesis, and is also involved in vascular development. MEF2C plays important role in emotional communication (45) mutations found in individuals with MCHS disrupt the DNA-binding function of MEF2C, and DNA-binding-deficient Mef2c global heterozygous mice display numerous MCHS-related phenotypes, including excitatory neuron and microglia gene expression changes. MEF2C regulates typical brain development and function through multiple cell types, including excitatory neuronal and neuroimmune populations (46).

Another study shows that MEF2C single-nucleotide polymorphisms are associated with schizophrenia, autism, and intellectual disability (47,48). However, there is a crucial role for MEF2C in programming early neuronal differentiation and proper distribution within the layers of the neocortex (49), and it was reported that deletion may cause severe mental retardation, seizures, hypotonia (50), and atypical Rett syndrome is due to the involvement of MEF2C in the common pathway (51).

\section{STAT3}

STATs (signal transducers and activators of transcription) are a family of seven transcription factors that form part of the JAK-STAT signaling cascade, the basis of the signal transduction mechanism for many cytokine receptors. STATs are activated by phosphorylation by JAKs. Although, the critical role in emotional interaction (52), a study show linkage between STAT3 and autism by STAT3 activation using luteolin, a citrus bioflavonoid, and its structural analog, diosmin, on IL-6 induced JAK2/STAT3 (Janus tyrosine kinase-2/signal transducer and activator of transcription-3) phosphorylation and signaling as well as behavioral phenotypes of MIA offspring (53-55). 
$\mathrm{Nr} 4 \mathrm{a} 2$, a transcription factor critical in cocaine-associated behaviors and necessary for MHb development, is enriched in the cholinergic cell-population of the $\mathrm{MHb}$; yet, the role of NR4A2 within the MHb in the adult brain remains elusive (56).NR4A2 was reported as a controller of emotions(57). Moreover, Another study reports language impairment, developmental delay, intellectual disability, autism spectrum disorder, early-onset dystonia-parkinsonism, and seizures $(58-61)$

\section{Conclusions}

Based on the performed in silico analysis, it was possible to identify the occurrence of specific motifs in the SARS-COV-2. These motifs could be able to bind specific host (human) TFs, and, consequently, recruitment/subtraction could be provoked. In this manner, as we hypothesized, a post-infection depression or anxiety effect is occurring, with consequences on involved target genes which are predicted by our in silico approach. Even though many studies are concentrated on the molecular mechanism of SARS-COV-2 infection, the exact mechanisms that induce the complex psychological symptomatology, including fear and depression for the patient and its family, remain unclear. An intricate network of TFs has been suggested by our analysis (Figure3). Interestingly, the functional annotation of putative target genes revealed evidence that TFs are specifically enriched in biological networks involved in different biological processes and especially in behavioral processes. All of these observations lead to a new insight, which should make possible the explanation of the complex behavioral and psychological interaction in SARS-COV-2 patients. Indeed, this in silico analysis showed evidence that different TFs can regulate multiple target genes, and it provides original predictive computational data, paving the way as a testable hypothesis to further studies. The SARS-COV- 2 remains ambiguous in its role in the development of fear mood state in the patients. Our data is suggesting, according to recent observations, that anxiety and fear feelings could be a result of the combined TFs, able to produce a negative effect on target genes that provoke this condition.

\section{Declarations}

\section{Compliance with ethical standards}

Conflict of interest There are no conflicts of interest to declare.

\section{References}

1. Wang J. Fast Identification of Possible Drug Treatment of Coronavirus Disease-19 (COVID-19) through Computational Drug Repurposing Study. Journal of Chemical Information and Modeling [Internet]. 2020 Jun 22 [cited 2021 Mar 27];60(6):3277-86. Available from:

https://pubs.acs.org/sharingguidelines

2. Elmezayen AD, Al-Obaidi A, Şahin AT, Yelekçi K. Drug repurposing for coronavirus (COVID-19): in silico screening of known drugs against coronavirus 3CL hydrolase and protease enzymes. Journal of Biomolecular Structure and Dynamics. 2020;1-13.

3. Senanayake SL. Drug repurposing strategies for COVID-19. Future Drug Discovery [Internet]. 2020 Apr 1 [cited 2021 Mar 27];2(2). Available from: https://clinicaltrials.gov/ct2/show/NCT04255017

4. Habib PT, Saber-Ayad M. In Silico Analysis of 716 Natural Bioactive Molecules Form Atlantic Ocean Reveals Candidate Molecule to Inhibit Spike Protein. 2021 [cited 2021 Mar 27]; Available from: https://doi.org/10.21203/rs.3.rs-156623/v1

5. Habib PT, Alsamman AM, Saber-Ayad M, Hassanein SE, Hamwieh A. COVIDier: A Deep-learning Tool For Coronaviruses Genome And Virulence Proteins Classification. biorxiv.org [Internet]. [cited 2021 Mar 27]; Available from: https://doi.org/10.1101/2020.05.03.075549

6. Habib PT. COVATOR: A Software for Chimeric Coronavirus Identification. biorxiv.org [Internet]. [cited $2021 \mathrm{Mar} 27$ ]; Available from: https://doi.org/10.1101/2020.11.14.383075

7. Alimadadi A, Aryal S, Manandhar I, Munroe PB, Joe B, Cheng X. Artificial intelligence and machine learning to fight covid-19. Vol. 52, Physiological Genomics. American Physiological Society; 2020. p. 200-2.

8. Yao H, Zhang N, Zhang R, Duan M, Xie T, Pan J, et al. Severity Detection for the Coronavirus Disease 2019 (COVID-19) Patients Using a Machine Learning Model Based on the Blood and Urine Tests. Frontiers in Cell and Developmental Biology. 2020 Jul 31;8.

9. Barstugan M, Ozkaya U, Ozturk S. Coronavirus (COVID-19) Classification using CT Images by Machine Learning Methods. arXiv. arXiv; 2020.

10. Tahir ul Qamar M, Rehman A, Tusleem K, Ashfaq UA, Qasim M, Zhu X, et al. Designing of a next generation multiepitope based vaccine (MEV) against SARS-COV-2: Immunoinformatics and in silico approaches. PLoS ONE. 2020 Dec 1;15(12 December 2020).

11. Abraham Peele K, Srihansa T, Krupanidhi S, Ayyagari VS, Venkateswarulu TC. Design of multi-epitope vaccine candidate against SARS-CoV-2: a insilico study. Journal of Biomolecular Structure and Dynamics. 2020;1-9.

12. Enayatkhani M, Hasaniazad M, Faezi S, Guklani H, Davoodian P, Ahmadi N, et al. Reverse vaccinology approach to design a novel multi-epitope vaccine candidate against COVID-19: an in silico study. Journal of Biomolecular Structure and Dynamics. 2020;

13. Habib PT. Vaccine Design, Adaptation, and Cloning Design for Multiple Epitope-Based Vaccine Derived From SARS-CoV-2 Surface Glycoprotein (S), Membrane Protein (M) and Envelope Protein (E): In silico approach. 2021 [cited 2021 Mar 27]; Available from: https://doi.org/10.21203/rs.3.rs-241638/v1

14. Tandon R. The COVID-19 pandemic, personal reflections on editorial responsibility. Vol. 50, Asian Journal of Psychiatry. Elsevier B.V.; 2020.

15. Tandon R. COVID-19 and mental health: Preserving humanity, maintaining sanity, and promoting health. Vol. 51, Asian Journal of Psychiatry. Elsevier B.V.; 2020. 
16. Sahoo S, Mehra A, Dua D, Suri V, Malhotra P, Yaddanapudi LN, et al. Psychological experience of patients admitted with SARS-CoV-2 infection [Internet]. Vol. 54, Asian Journal of Psychiatry. Elsevier B.V.; 2020 [cited 2021 Mar 27]. p. 102355. Available from: /pmc/articles/PMC7434329/

17. Wang C, Pan R, Wan X, Tan Y, Xu L, Ho CS, et al. Immediate psychological responses and associated factors during the initial stage of the 2019 coronavirus disease (COVID-19) epidemic among the general population in China. International Journal of Environmental Research and Public Health. 2020 Mar 1;17(5).

18. Huang Y, Zhao N. Chinese mental health burden during the COVID-19 pandemic. Vol. 51, Asian Journal of Psychiatry. Elsevier B.V.; 2020.

19. BioScience PH-H in, 2021 undefined. COVID-19 symphony: A review of possible music therapy effect in supporting the immune system of COVID-19 patient. highlightsin.org [Internet]. [cited 2021 Mar 27]; Available from: http://www.highlightsin.org/index.php/bioscience/article/view/104

20. Kang L, Li Y, Hu S, Chen M, Yang C, Yang BX, et al. The mental health of medical workers in Wuhan, China dealing with the 2019 novel coronavirus. Vol. 7, The Lancet Psychiatry. Elsevier Ltd; 2020. p. e14.

21. Lai J, Ma S, Wang Y, Cai Z, Hu J, Wei N, et al. Factors Associated With Mental Health Outcomes Among Health Care Workers Exposed to Coronavirus Disease 2019. JAMA network open. 2020 Mar 2;3(3):e203976.

22. Rossi R, Socci V, Pacitti F, di Lorenzo G, di Marco A, Siracusano A, et al. Mental health outcomes among front and second line health workers associated with the COVID-19 pandemic in Italy. medRxiv. medRxiv; 2020.

23. Tan BYQ, Chew NWS, Lee GKH, Jing M, Goh Y, Yeo LLL, et al. Psychological Impact of the COVID-19 Pandemic on Health Care Workers in Singapore. Vol. 173, Annals of internal medicine. NLM (Medline); 2020. p. 317-20.

24. Grover S, Dua D, Sahoo S, Mehra A, Nehra R, Chakrabarti S. Why all COVID-19 hospitals should have mental health professionals: The importance of mental health in a worldwide crisis! Asian Journal of Psychiatry. 2020 Jun 1;51.

25. Yao H, Chen JH, Xu YF. Patients with mental health disorders in the COVID-19 epidemic. Vol. 7, The Lancet Psychiatry. Elsevier Ltd; 2020. p. e21.

26. Rogers JP, Chesney E, Oliver D, Pollak TA, McGuire P, Fusar-Poli P, et al. Psychiatric and neuropsychiatric presentations associated with severe coronavirus infections: a systematic review and meta-analysis with comparison to the COVID-19 pandemic. The Lancet Psychiatry. 2020 Jul 1;7(7):61127.

27. Chen N, Zhou M, Dong X, Qu J, Gong F, Han Y, et al. Epidemiological and clinical characteristics of 99 cases of 2019 novel coronavirus pneumonia in Wuhan, China: a descriptive study. The Lancet. 2020 Feb 15;395(10223):507-13.

28. Helms J, Kremer S, Merdji H, Clere-Jehl R, Schenck M, Kummerlen C, et al. Neurologic Features in Severe SARS-CoV-2 Infection. New England Journal of Medicine. 2020 Jun 4;382(23):2268-70.

29. Del'Guidice T, Latapy C, ... AR-P of the, 2015 undefined. FXR1P is a GSK3 $\beta$ substrate regulating mood and emotion processing. National Acad Sciences [Internet]. [cited 2021 Mar 27]; Available from: https://www.pnas.org/content/112/33/E4610.short

30. Lee SA, Tsao TT, Yang KC, Lin H, Kuo YL, Hsu CH, et al. Construction and analysis of the protein-protein interaction networks for schizophrenia, bipolar disorder, and major depression. BMC Bioinformatics. 2011 Nov 30;12(SUPPL. 13).

31. Blank T, Detje C, Spieß A, Immunity NH-, 2016 undefined. Brain endothelial-and epithelial-specific interferon receptor chain 1 drives virus-induced sickness behavior and cognitive impairment. Elsevier [Internet]. [cited 2021 Mar 27]; Available from:

https://www.sciencedirect.com/science/article/pii/S1074761316301108

32. Zhang J, Lu H, Zeng H, Zhang S, Du Q, Jiang T, et al. The differential psychological distress of populations affected by the COVID-19 pandemic. Vol. 87, Brain, Behavior, and Immunity. Academic Press Inc.; 2020. p. 49-50.

33. Bo HX, Li W, Yang Y, Wang Y, Zhang Q, Cheung T, et al. Posttraumatic stress symptoms and attitude toward crisis mental health services among clinically stable patients with COVID-19 in China. Psychological Medicine. 2020;

34. Chetta M, di Pietro L, Bukvic N, Lattanzi W. Rising roles of small noncoding RNAs in cotranscriptional regulation: In silico study of miRNA and piRNA regulatory network in humans. Genes. 2020 May 1;11(5).

35. Gupta S, Stamatoyannopoulos JA, Bailey TL, Noble WS. Quantifying similarity between motifs. Genome Biology. 2007 Feb 26;8(2).

36. Kuhn M, von Mering C, Campillos M, Jensen LJ, Bork P. STITCH: Interaction networks of chemicals and proteins. Nucleic Acids Research. 2008 Jan;36(SUPPL. 1)

37. Gaub S, Fisher SE, Ehret G. Ultrasonic vocalizations of adult male Foxp2-mutant mice: behavioral contexts of arousal and emotion. Genes, Brain and Behavior [Internet]. 2016 Feb 1 [cited 2021 Mar 27];15(2):243-59. Available from: https://onlinelibrary.wiley.com/doi/abs/10.1111/gbb.12274

38. McCarthy-Jones S, Green M, ... RS-J of psychiatric, 2014 undefined. Preliminary evidence of an interaction between the FOXP2 gene and childhood emotional abuse predicting likelihood of auditory verbal hallucinations in. Elsevier [Internet]. [cited 2021 Mar 27]; Available from: https://www.sciencedirect.com/science/article/pii/S0022395613003646

39. Co M, Hickey SL, Kulkarni A, Harper M, Konopka G. Cortical Foxp2 supports behavioral flexibility and developmental dopamine D1 receptor expression. biorxiv.org [Internet]. [cited 2021 Mar 27]; Available from: https://doi.org/10.1101/624973

40. Heston JB, White SA. Behavior-Linked FoxP2 Regulation Enables Zebra Finch Vocal Learning. Soc Neuroscience [Internet]. 2015 [cited 2021 Mar 27 ]; Available from: https://www.jneurosci.org/content/35/7/2885.short

41. Medvedeva V, Rieger M, ... BV-H molecular, 2019 undefined. Altered social behavior in mice carrying a cortical Foxp2 deletion. academic.oup.com [Internet]. [cited 2021 Mar 27]; Available from: https://academic.oup.com/hmg/article-abstract/28/5/701/5142927

42. Chabout J, Sarkar A, Patel SR, Radden T, Dunson DB, Fisher SE, et al. A Foxp2 mutation implicated in human speech deficits alters sequencing of ultrasonic vocalizations in adult male mice. Frontiers in Behavioral Neuroscience. 2016 Oct 20;10(OCT). 
43. French C, Veloz M, Zhou K, Peter S, ... SF-M, 2019 undefined. Differential effects of Foxp2 disruption in distinct motor circuits. nature.com [Internet]. [cited 2021 Mar 27]; Available from: https://www.nature.com/articles/s41380-018-0199-x

44. Designed Research; S XGX, Chen Y, Chen Y, Yiwei Cao HZ, Performed Research; B XXL, Pnas | JD. Foxp2 regulates anatomical features that may be relevant for vocal behaviors and bipedal locomotion. National Acad Sciences [Internet]. 2018 [cited 2021 Mar 27];115(35):8799-804. Available from: https://www.pnas.org/content/115/35/8799.short

45. Carhuatanta KAK, Shea CJA, Herman JP, Jankord R. Unique genetic loci identified for emotional behavior in control and chronic stress conditions. Frontiers in Behavioral Neuroscience. 2014 Oct 21;8(OCT).

46. Harrington AJ, Bridges CM, Berto S, Blankenship K, Cho JY, Assali A, et al. MEF2C Hypofunction in Neuronal and Neuroimmune Populations Produces MEF2C Haploinsufficiency Syndrome-like Behaviors in Mice. Biological Psychiatry. 2020 Sep 15;88(6):488-99.

47. Mitchell A, Javidfar B, Pothula V, Ibi D, ... ES-M, 2018 undefined. MEF2C transcription factor is associated with the genetic and epigenetic risk architecture of schizophrenia and improves cognition in mice. nature.com [Internet]. [cited 2021 Mar 27]; Available from:

https://idp.nature.com/authorize/casa?

redirect_uri=https://www.nature.com/articles/mp2016254\&casa_token=5_MS70xODfQAAAAA:jzT2P_1mjx6E3e81nQW9KjjXv2NnjTC7RMEp6U8ujYp_Yu2 wvdjcvq7C5EaevrukXb47ceEDpYQ_OQ

48. Harrington AJ, Raissi A, Rajkovich K, Berto S, Kumar J, Molinaro G, et al. MEF2C regulates cortical inhibitory and excitatory synapses and behaviors relevant to neurodevelopmental disorders. elifesciences.org [Internet]. 2016 [cited 2021 Mar 27]; Available from: https://elifesciences.org/articles/20059

49. Li H, Radford JC, Ragusa MJ, Shea KL, Mckercher SR, Zaremba JD, et al. Transcription factor MEF2C influences neural stem/progenitor cell differentiation and maturation in vivo [Internet]. National Acad Sciences. 2008 [cited 2021 Mar 27]. Available from: www.pnas.org/cgi/content/full/

50. Nowakowska BA, Obersztyn E, Szyma nska K, Bekiesi nska-Figatowska M, Xia Z, Ricks CB, et al. Severe Mental Retardation, Seizures, and Hypotonia Due to Deletions of MEF2C Division of Genetic and Metabolic Disorders. Wiley Online Library [Internet]. 2010 [cited 2021 Mar 27];153(5):1042-51. Available from: www.interscience.wiley.com

51. Zweier M, Gregor A, Zweier C, Engels H, Sticht H, Wohlleber E, et al. Human Mutation RESEARCH ARTICLE Mutations in MEF2C from the 5q14.3q15 Microdeletion Syndrome Region Are a Frequent Cause of Severe Mental Retardation and Diminish MECP2 and CDKL5 Expression. North West Thames Regional Genetics Service, North West London Hospitals NHS Trust [Internet]. 2010 [cited 2021 Mar 27];5(6):722-33. Available from: www.interscience.wiley.com

52. Reisinger S, Sideromenos S, ... OH-M, 2020 undefined. STAT3 in the dorsal raphe gates behavioural reactivity and regulates gene networks associated with psychopathology. nature.com [Internet]. [cited 2021 Mar 27]; Available from: https://www.nature.com/articles/s41380-020-00904-2

53. Kong E, Sucic S, Monje F, Reisinger S, reports GS-S, 2015 undefined. STAT3 controls IL6-dependent regulation of serotonin transporter function and depression-like behavior. nature.com [Internet]. [cited 2021 Mar 27]; Available from: https://www.nature.com/articles/srep09009

54. Liu W, Liu J, Xia J, Xue X, Wang H, Qi Z, et al. Leptin receptor knockout-induced depression-like behaviors and attenuated antidepressant effects of exercise are associated with STAT3/SOCS3 signaling. Elsevier [Internet]. [cited 2021 Mar 27]; Available from:

https://www.sciencedirect.com/science/article/pii/S0889159117300016

55. Parker-Athill E, Luo D, Bailey A, Giunta B, ... JT-J of, 2009 undefined. Flavonoids, a prenatal prophylaxis via targeting JAK2/STAT3 signaling to oppose IL-6/MIA associated autism. Elsevier [Internet]. [cited 2021 Mar 27]; Available from:

https://www.sciencedirect.com/science/article/pii/S0165572809003166

56. López A, Hemstedt T, Jia Y, ... PH-, 2019 undefined. Epigenetic regulation of immediate-early gene Nr4a2/Nurr1 in the medial habenula during reinstatement of cocaine-associated behavior. Elsevier [Internet]. [cited 2021 Mar 27]; Available from:

https://www.sciencedirect.com/science/article/pii/S0028390819301339

57. Guo YP, Zhi YR, Liu TT, Wang Y, Zhang Y. Global gene knockout of kcnip3 enhances pain sensitivity and exacerbates negative emotions in rats. Frontiers in Molecular Neuroscience. 2019 Jan 24;12.

58. Lévy J, Grotto | S, Mignot | C, Maruani | A, Delahaye-Duriez | A, Benzacken | B, et al. NR4A2 haploinsufficiency is associated with intellectual disability and autism spectrum disorder. Wiley Online Library [Internet]. 2018 Aug 1 [cited 2021 Mar 27];94(2):264-8. Available from:

https://onlinelibrary.wiley.com/doi/abs/10.1111/cge.13383

59. Jesús S, Hinarejos I, Carrillo F, Martínez-Rubio D, Macías-García D, Sánchez-Monteagudo A, et al. NR4A2 Mutations Can Cause Intellectual Disability and Language Impairment With Persistent Dystonia-Parkinsonism MORE ONLINE Video. Neurol Genet [Internet]. 2021 [cited 2021 Mar 27];7:543. Available from: https://ng.neurology.org/content/7/1/e543.abstract

60. Singh S, Gupta A, Zech M, Sigafoos A, ... KC-G in, 2020 undefined. De novo variants of NR4A2 are associated with neurodevelopmental disorder and epilepsy. nature.com [Internet]. [cited 2021 Mar 27]; Available from: https://www.nature.com/articles/s41436-020-0815-4

61. Verhelst H, Verloo P, ... AD-EJ of, 2017 undefined. NR4A2 causes an autism spectrum disorder. ejpn-journal.com [Internet]. [cited 2021 Mar 27]; Available from: https://www.ejpn-journal.com/article/S1090-3798(17)31148-0/fulltext

\section{Figures}




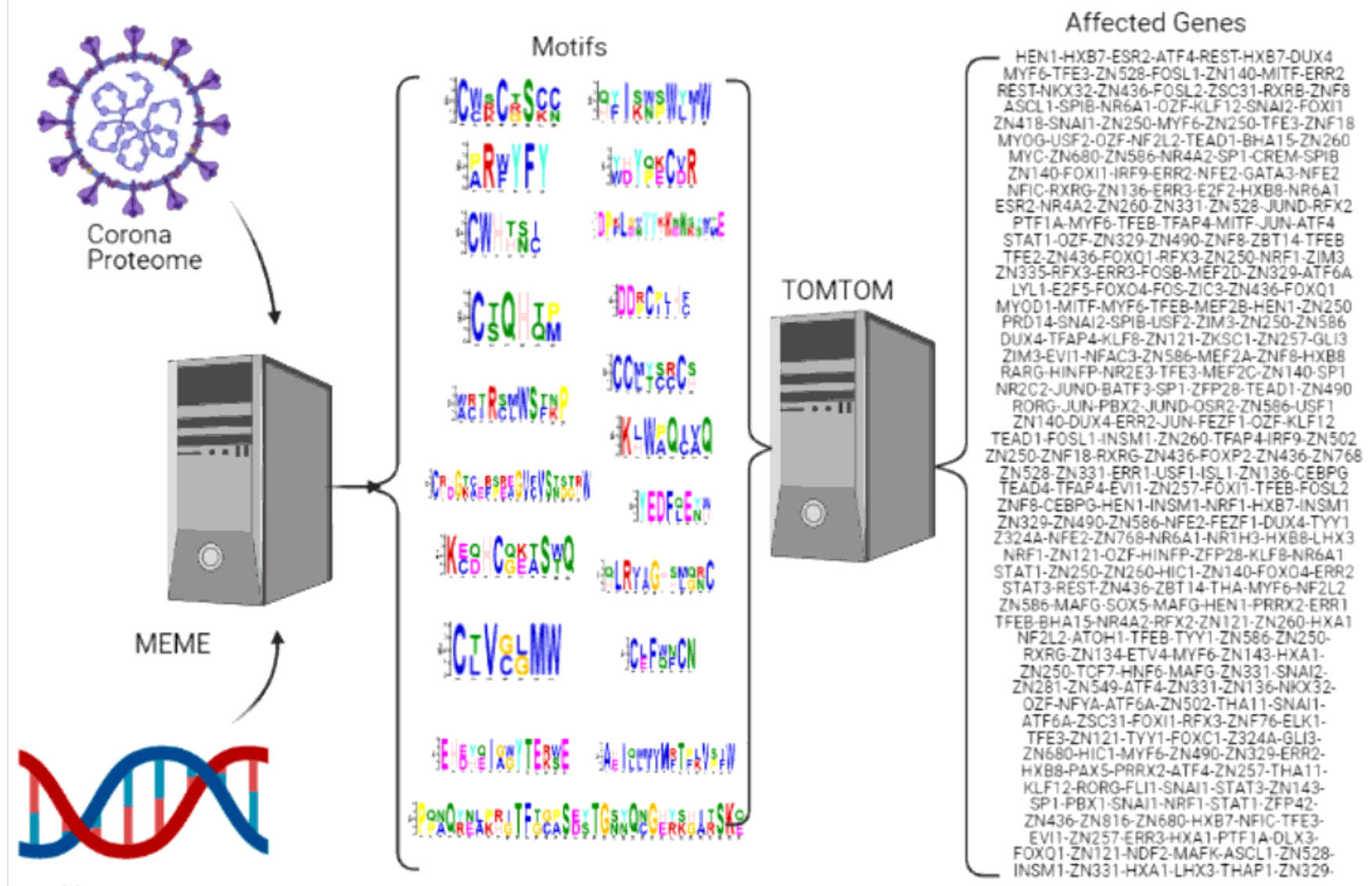

Human

Genome

Figure 1

Workflow of SARS-COV-2 motifs Analysis 


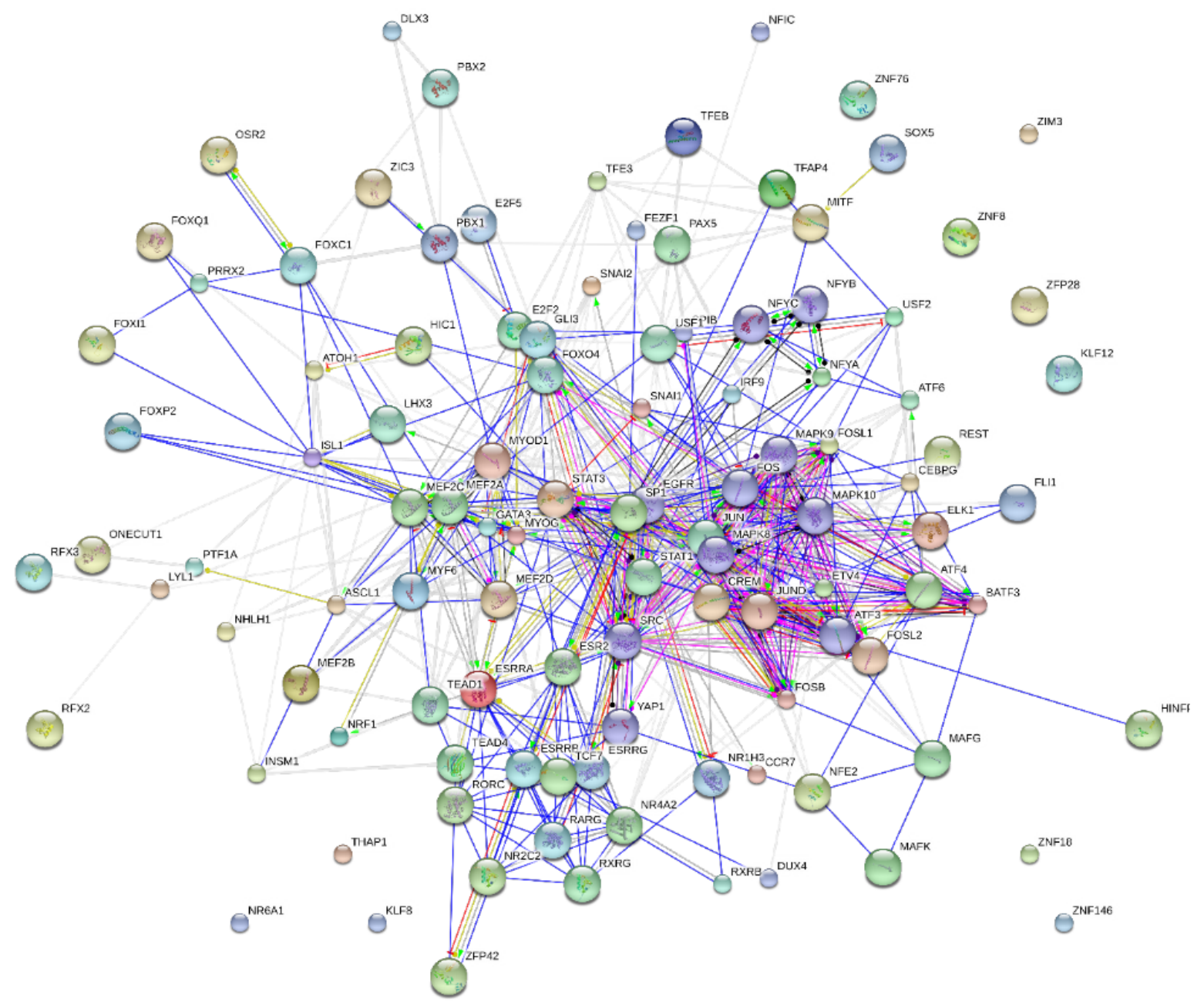

Figure 2

Network analysis shows the affected genes with transcription factors 


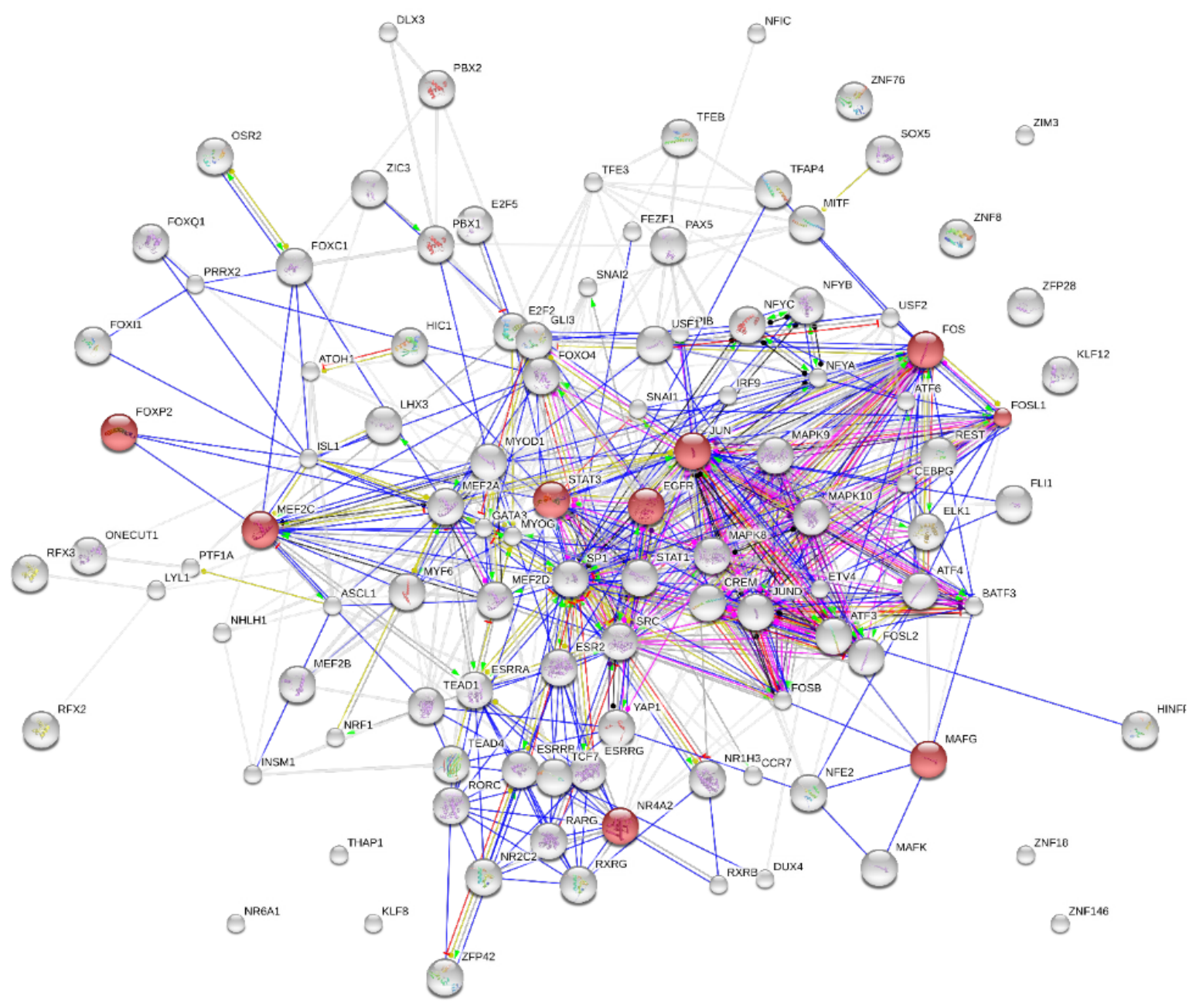

Figure 3

Genes responsible of behavioral and mental states (Red Nodes) 